\title{
Hydrothermal Synthesis and Structure of Dihydrated Cobalt Pyrophosphates
}

\author{
Ganjam S. GopalaKrishna, * Madegowda MAHEndra, ** Beeranahally H. Doreswamy, ** \\ Madagudanahalli J. MAHESH, ${ }^{*}$ Sridhar M. ANANDALWAR, ${ }^{* *}$ and Javaregowda S. PraSAD ${ }^{* * *}$ \\ *Department of Studies in Geology, University of Mysore, Manasagangotri, Mysore 570 006, India \\ **Department of Studies in Physics, University of Mysore, Manasagangotri, Mysore 570 006, India
}

\begin{abstract}
$\mathrm{H}_{2} \mathrm{Co}_{2} \mathrm{P}_{4} \mathrm{O}_{14}$ crystals were synthesized by a hydrothermal technique. They crystallized in a monoclinic system with cell parameters $a=9.1609(7) \AA, b=12.6764(8) \AA, c=9.6868(10) \AA$ and $\beta=106.771(7)^{\circ}$. An open framework structure having tunnel-type cavities is observed.
\end{abstract}

(Received November 5, 2004; Accepted April 5, 2004; Published on Web June 16, 2004)

Phosphates were studied extensively owing to their wide range of applications such as piezoelectric, magnetic, luminescence, ceramics and superionics application. ${ }^{1-3}$ Subsequently, there were also reports on pyrophosphates exhibiting simple framework structure with relatively high ionic conductivity values. $^{4}$ Recently, there are also reports on hydrated phosphates. ${ }^{5}$ Here, we report a new group of hydrated cobaltbearing pyrophosphates, which is paramagnetic in nature at ambient condition.

$\mathrm{H}_{2} \mathrm{Co}_{2} \mathrm{P}_{4} \mathrm{O}_{14}$ crystals were synthesized by hydrothermal processing. The growth of phosphates by the hydrothermal technique is relatively new and is quite complicated for these compounds, because of the highly corrosive and volatile nature of phosphorus at higher temperature. Following earlier methods, the present experiments were carried out in Morey type autoclaves provided with Teflon liners, in the temperature and pressure range of $230-265^{\circ} \mathrm{C}$ and $60-100$ bars. All reagents used in the synthesis process were of Analar grade with 99.9\% purity from Merck. The desired quantity of $\mathrm{CoCl}_{3}$ was dissolved in $10 \mathrm{ml}$ of $0.25 \mathrm{M}$ of ETDA solution and the mixture was taken in Teflon liners, then $6 \mathrm{ml}$ of $85 \% \mathrm{H}_{3} \mathrm{PO}_{4}$ was added. The crystallization was carried out by spontaneous nucleation controlled through a slow and programmed rate of heating. The authors were able to synthesize $\mathrm{H}_{2} \mathrm{Co}_{2} \mathrm{P}_{4} \mathrm{O}_{14}$ crystals under the following experimental conditions: molar ratio, $\mathrm{Co}_{2} \mathrm{O}_{3}: \mathrm{P}_{2} \mathrm{O}_{5}$ : $\mathrm{H}_{2} \mathrm{O}=1-1.5: 10-12: 8-10 ; T, 260^{\circ} \mathrm{C} ; P, 80$ bars; duration $=$ 8 - 10 days.

The following reaction could explain the formation of $\mathrm{H}_{2} \mathrm{Co}_{2} \mathrm{P}_{4} \mathrm{O}_{14}$ :

$$
2 \mathrm{CoCl}_{3}+4 \mathrm{H}_{3} \mathrm{PO}_{4}+2 \mathrm{H}_{2} \mathrm{O} \rightarrow \mathrm{H}_{2} \mathrm{Co}_{2} \mathrm{P}_{4} \mathrm{O}_{14}+6 \mathrm{HCl}+4 \mathrm{H}_{2} \mathrm{O}
$$

The crystals obtained by this method were of good quality, exhibiting translucent luster with well developed morphology. The sizes of crystals range from 0.5 to $3 \mathrm{~mm}$ with pink color.

A single crystal of $\mathrm{H}_{2} \mathrm{Co}_{2} \mathrm{P}_{4} \mathrm{O}_{14}$ of dimensions $0.2 \times 0.2 \times 0.2$ $\mathrm{mm}$ was chosen for X-ray diffraction studies. The crystal and experimental data are given in Table 1. The structure was solved by direct methods and refined by full-matrix least

† To whom correspondence should be addressed.

E-mail: jsp@uomphysics.net squares methods with anisotropic temperature factors for the non-H atoms. $\mathrm{H}$ atoms were generated by a difference Fourier map and were placed at a distance of $0.98 \AA$ from the parent atom. The maximum peak lies at the nearest distances of 0.76 $\AA, 1.58 \AA, 1.85 \AA$ and $1.92 \AA$ from the atoms Co1, O3, O11 and O5, respectively. The final coordinates and equivalent thermal parameters of non- $\mathrm{H}$ atoms are listed in Table 2. Table 3 gives selected bond distances and bond angles; they agree with their standard values. Figure 1 represents the ORTEP diagram of the molecule with thermal ellipsoids at $50 \%$ probability. Crystal structure has an additional feature of a psuedo $I$-centred lattice translation of $(0.5,0.5,0.5)$ for all the atoms except $\mathrm{O} 7, \mathrm{O} 10$, O16 and O19, respectively. But the structure could not be solved and refined in the space group I2/a.

The polymerization of phosphate with Co has revealed its coordination and packing mode. Each $\mathrm{Co}$ atom has coordination with six pyrophosphate oxygen atoms i.e., from two O-atoms of one terminal pyrophosphate. This coordination environment resembles a distorted hexahedron representation or six membered ring column. Also, the $\mathrm{Co}-\mathrm{O}-\mathrm{Co}-\mathrm{O}$ ring

Table 1 Crystal and experimental data

\begin{tabular}{ll}
\hline Formula & $\mathrm{H}_{2} \mathrm{Co}_{2} \mathrm{O}_{14} \mathrm{P}_{4}$ \\
Formula weight & 467.76 \\
Crystal system & monoclinic \\
Space group & $P 2_{1} / c, \mathrm{Z}=4$ \\
$a$ & $9.1609(7) \AA$ \\
$b$ & $12.6764(8) \AA$ \\
$c$ & $9.6868(10) \AA$ \\
$\beta$ & $106.771(7) \AA$ \\
$V$ & $1077.06(15) \AA^{3}$ \\
$D_{x}$ & $2.885 \mathrm{Mg} / \mathrm{cm}^{3}$ \\
$\theta_{\max }$ & $30.02^{\circ}$ with Mo $K_{\alpha}$ \\
$R$ & 0.0382 \\
$(\Delta / \sigma)_{\max }$ & 0.001 \\
$(\Delta \rho)_{\max }$ & $1.111 \mathrm{e} \AA^{-3}$ \\
$(\Delta \rho)_{\min }$ & $-1.093 \mathrm{e} \AA^{-3}$ \\
Measurement & DipLabo Kappa \\
Program system & Denzo \\
Structure determination & SHELXS-97 \\
Refinement & fullmatrix: SHELXL-97 \\
\hline
\end{tabular}


Table 2 Atomic coordinates and equivalent temperature factors $\left(\AA^{2}\right)$

\begin{tabular}{lrrrr}
\hline Atom & \multicolumn{1}{c}{$x$} & \multicolumn{1}{c}{$y$} & & \\
\hline Co1 & $0.75030(3)$ & $0.25490(3)$ & $-0.24969(3)$ & $0.00847(13)$ \\
Co2 & $0.74781(4)$ & $0.08553(3)$ & $-0.00442(3)$ & $0.00884(13)$ \\
O3 & $0.6188(2)$ & $0.21742(15)$ & $-0.1140(2)$ & $0.0114(4)$ \\
P4 & $0.51443(8)$ & $0.29103(6)$ & $-0.06417(7)$ & $0.01059(17)$ \\
O5 & $0.5638(3)$ & $0.31532(17)$ & $0.0935(2)$ & $0.0170(4)$ \\
O6 & $0.3494(3)$ & $0.2478(3)$ & $-0.1042(3)$ & $0.0414(8)$ \\
O7 & $0.5025(4)$ & $0.3980(2)$ & $-0.1468(3)$ & $0.0475(10)$ \\
P8 & $0.54163(8)$ & $0.03871(6)$ & $0.22708(7)$ & $0.01098(17)$ \\
O9 & $0.6025(2)$ & $-0.02151(17)$ & $-0.1125(2)$ & $0.0201(5)$ \\
O10 & $0.6158(3)$ & $-0.0646(2)$ & $0.3013(3)$ & $0.0294(6)$ \\
O11 & $0.6626(2)$ & $0.09934(15)$ & $0.1822(2)$ & $0.0128(4)$ \\
O12 & $0.8835(2)$ & $0.20750(15)$ & $0.1142(2)$ & $0.0107(4)$ \\
P13 & $0.99318(8)$ & $0.27895(5)$ & $0.06757(7)$ & $0.00888(16)$ \\
O14 & $0.9303(3)$ & $0.17526(16)$ & $0.4196(2)$ & $0.0162(4)$ \\
O15 & $1.1502(3)$ & $0.22399(19)$ & $0.0886(3)$ & $0.0209(5)$ \\
O16 & $1.0462(2)$ & $0.36873(17)$ & $0.1838(2)$ & $0.0178(4)$ \\
P17 & $0.97722(8)$ & $0.44506(5)$ & $0.28156(7)$ & $0.00920(16)$ \\
O18 & $1.1084(2)$ & $0.46732(17)$ & $0.4108(2)$ & $0.0169(4)$ \\
O19 & $0.9113(3)$ & $0.54082(18)$ & $0.1868(3)$ & $0.0237(5)$ \\
O20 & $0.8469(2)$ & $0.11116(15)$ & $-0.1829(2)$ & $0.0117(4)$ \\
\hline
\end{tabular}

$U_{\mathrm{eq}}=(1 / 3) \sum_{i} \Sigma_{j} U_{i j}\left(a_{i}^{*} a_{j}^{*}\right)\left(\boldsymbol{a}_{i} \cdot \boldsymbol{a}_{j}\right)$.

Table 3 Bond lengths $(\AA)$ and bond angles $\left({ }^{\circ}\right)$

\begin{tabular}{lrlr}
\hline \multicolumn{1}{c}{ Atoms } & Length & \multicolumn{1}{c}{ Atoms } & \multicolumn{1}{c}{ Length } \\
\hline P17-O18 & $1.491(2)$ & Co2-O3 & $2.1434(19)$ \\
P17-O20\#e & $1.513(2)$ & Co2-O18\#a & $2.028(2)$ \\
P4-O5 & $1.494(2)$ & Co1-O20 & $2.047(2)$ \\
P4-O6 & $1.549(3)$ & Co1-O12\#d & $2.094(2)$ \\
P8-O9\#c & $1.475(2)$ & Co1-O14 & $2.152(2)$ \\
P8-O11 & $1.512(2)$ & O11-Co1\#e & $2.047(2)$ \\
P13-O12 & $1.515(2)$ & O14-P13\#e & $1.499(2)$ \\
\hline $\mathrm{O}(18)-\mathrm{P}(17)-\mathrm{O}(20) \# \mathrm{e}$ & $113.32(12)$ & $\mathrm{O}(14) \# \mathrm{~d}-\mathrm{P}(13)-\mathrm{O}(12)$ & $114.32(12)$ \\
$\mathrm{O}(18)-\mathrm{P}(17)-\mathrm{O}(19)$ & $115.77(13$ & $\mathrm{O}(18) \# \mathrm{a}-\mathrm{Co}(2)-\mathrm{O}(20)$ & $95.66(8)$ \\
$\mathrm{O}(20) \# \mathrm{e}-\mathrm{P}(17)-\mathrm{O}(19)$ & $107.93(12)$ & $\mathrm{O}(11)-\mathrm{Co}(2)-\mathrm{O}(20)$ & $166.45(8)$ \\
$\mathrm{O}(18)-\mathrm{P}(17)-\mathrm{O}(16)$ & $104.51(12)$ & $\mathrm{O}(20)-\mathrm{Co}(1)-\mathrm{O}(12) \# \mathrm{~d}$ & $97.13(8)$ \\
$\mathrm{O}(20) \# \mathrm{e}-\mathrm{P}(17)-\mathrm{O}(16)$ & $108.71(11)$ & $\mathrm{P}(8)-\mathrm{O}(11)-\mathrm{Co}(2)$ & $131.87(12)$ \\
$\mathrm{O}(19)-\mathrm{P}(17)-\mathrm{O}(16)$ & $106.13(13)$ & $\mathrm{Co}(1) \# \mathrm{e}-\mathrm{O}(11)-\mathrm{Co}(2)$ & $97.99(8)$ \\
$\mathrm{O}(9) \# \mathrm{c}-\mathrm{P}(8)-\mathrm{O}(11)$ & $115.45(13)$ & $\mathrm{P}(17)-\mathrm{O}(18)-\mathrm{Co}(2) \# \mathrm{~b}$ & $141.01(14)$ \\
$\mathrm{O}(12)-\mathrm{P}(13)-\mathrm{O}(16)$ & $108.24(11)$ & $\mathrm{P}(13)-\mathrm{O}(12)-\mathrm{Co}(2)$ & $127.91(12)$ \\
\hline
\end{tabular}

represents a four membered ring column. These condensed phosphates have been extended linearly and interlinked via metal coordination, which in turn exhibits three dimensional open framework structure. Tunnel-type cavities are observed in the molecular packing diagram along the $a$ axis. By suitable

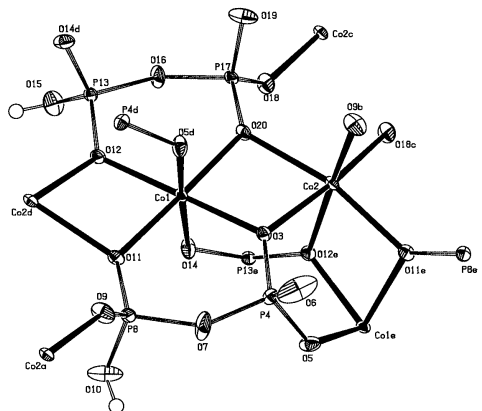

Fig. 1 ORTEP diagram of the molecule at $50 \%$ probability. Symmetry transformations used to generate equivalent atoms: \#a $(-x$ $+2, y-1 / 2,-z+1 / 2)$, \#b $(-x+2, y+1 / 2,-z+1 / 2)$, \#c $(-x+1,-y$, $-z)$, \#d $(x,-y+1 / 2, z-1 / 2)$, \#e $(x,-y+1 / 2, z+1 / 2)$.

modification in the synthetic procedure, we can have the participation of alkali metal ions in the cavity as expected for the ion transport mechanism. ${ }^{6}$

\section{Acknowledgements}

The authors would like to express their thanks to DST, Government of India for financial assistance under the project SP/I2/FOO/93.

\section{References}

1. H. P. Weber, T. C. Dmen, H. G. Daniel Meyer, and B. C. Tofield, Appl. Phys. Lett., 1973, 22, 534.

2. H. Y. P. Hong, Mater. Res. Bull., 1976, 11, 173.

3. M. Forsyth, S. Wong, K. M. Nairn, A. S. Best, P. J. Neman, and D. R. MacFarlane, Sol. State Ion., 1999, 124, 213.

4. N. K. Lokanath, M. A. Sridhar, J. Shashidhara Prasad, G. S. Gopalakrishna, and K. G. Ashamanjari, J. Mat. Sci. Lett., 1999, 18, 1723.

5. K. Byrappa, B. V. Umeshdutta, A. Clearfield, and D. Poojary, J. Mat. Res., 1994, 9, 1510.

6. M. Mahendra, S. P. Madhu, B. H. Doreswamy, G. S. Gopalakrishna, M. A. Sridhar, J. Shashidhara Prasad, and K. G. Ashamanjari, Mat. Res. Bull., 2003, 37, 1309. 